DOE/ER/60892--2

DE9 1007528

\section{SYNTHESIS AND IN-VIVO DETECTION OF BORONATED COMPOUNDS FOR USE IN BNCT}

Progress Report

\begin{abstract}
August 1, 1990 - July 31, 1991
George W. Kabalka, Ph.D.

Department of Radiology

University of Tennessee Medical Centei at Knoxville

Knoxville, Tennessee 37920
\end{abstract}

February 1991

February 199
- wis wil

FEB 141991

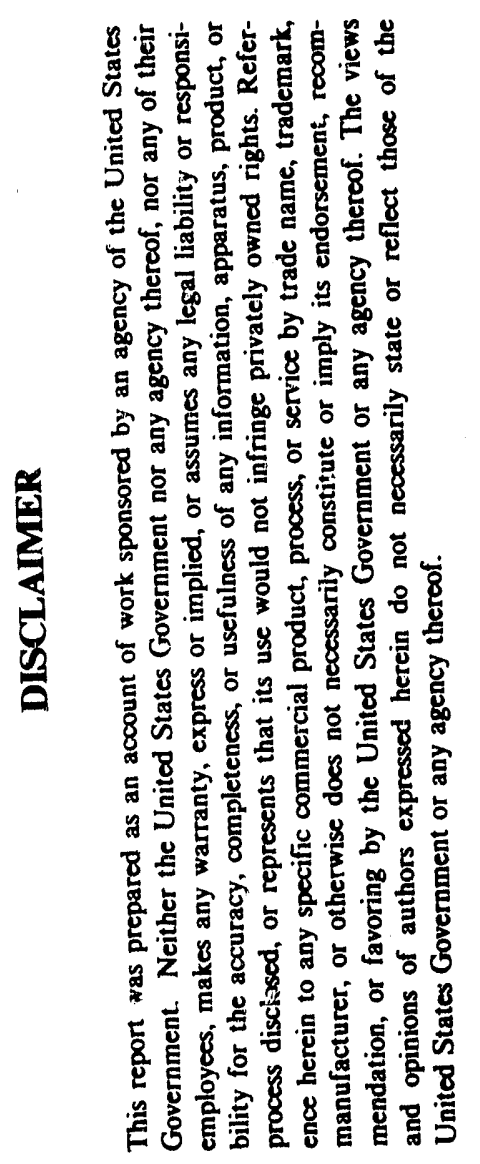

PREPARED FOR THE U.S. DEPARTMENT OF ENERGY UNDER GRANT NUMBER DE-FG05-89ER60892

\title{
MASTER
}




\section{TABLE OF CONTENTS}

\section{Section}

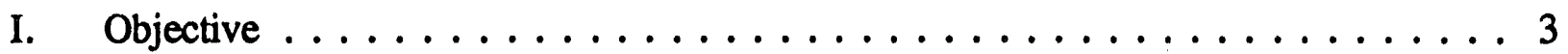

II. Research Accomplishments $\ldots \ldots \ldots \ldots \ldots \ldots \ldots \ldots \ldots \ldots$

III. Bibliography Of Publications $\ldots \ldots \ldots \ldots \ldots \ldots \ldots \ldots$

IV. Graduate and Postdoctoral Students $\ldots \ldots \ldots \ldots \ldots \ldots \ldots$

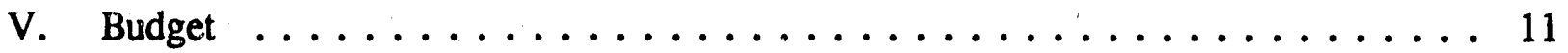




\section{OBJECTIVE}

The primary objectives of the D.O.E. Program at the University of Tennessee Biomedical Imaging Center are the development of new boron-neutron-capture agents as well as the technology to detect boron compounds in-vivo. The detection technology focuses on the development of effective magnetic resonance imaging (MRI) and spectroscopy (MRS) techniques for verifying and measuring BNCT agents in-vivo. A significant portion of the effort is directed toward the design of boron-containing neutron-capture-therapy agents. The U.T. - D.O.E. program is unique in that it has access to two state-of-the-art multinuclear magnetic resonance imaging units housed in the Biomedical Imaging Center at the University of Tennessee Medical Center at Knoxville. In addition the U.T. - D.O.E. researchers actively collaborate with colleagues at other D.O.E. facilities (Brookhaven National Laboratory, Oak Ridge National Laboratory, Los Alamos National Laboratory and Oak Ridge Associated Universities).

An important goal of the D.O.E. program at U.T. is to provide training for students (predoctoral and postdoctoral). The University of Tennessee is one of the very few institutions in the world where students have "hands-on" access to both modern scientific equipment and medical imaging modalities such as the clinical MRI units. The academic nature of the program facilitates collaborative interactions with other D.O.E. programs and helps to insure the continued availability of skilled scientists dedicated to the advancement of diagnostic medical procedures. 


\section{RESEARCH ACCOMPLISHMENTS}

I am pleased to report that this has been a productive year for the D.O.E. program at the University of Tennessee. Five journal articles have resulted from the work and nine scientific presentations were made; They are compiled in Section III.

\section{A. SYNTHESIS OF POTENTIAL NEUTRON CAPTURE THERAPY AGENTS}

\section{Carboranyl Precursors}

Carboranes contain ten boron atoms in a three-dimensional space equivalent to a benzene ring; consequently, the carborane isomers can be utilized to prepare a variety of boron-rich agents for potential use in boron-neutron-capture therapy.
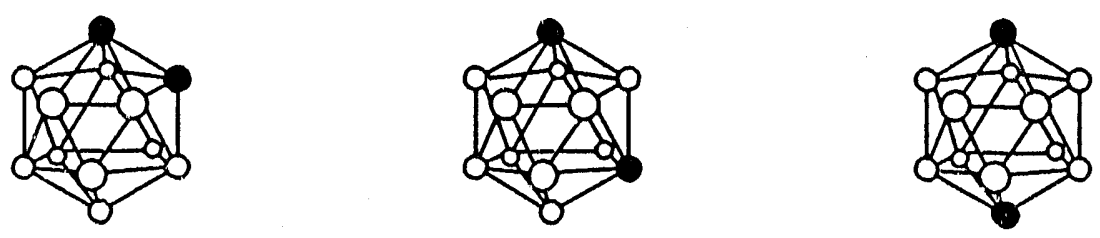

$$
\text { [Where } \mathrm{O}=\mathrm{BH}, \Theta=\mathrm{CH} \text { ] }
$$

We are currently developing synthetic methodology suitable for preparing amino acids and other physiologically active compounds of potential use in BNCT.
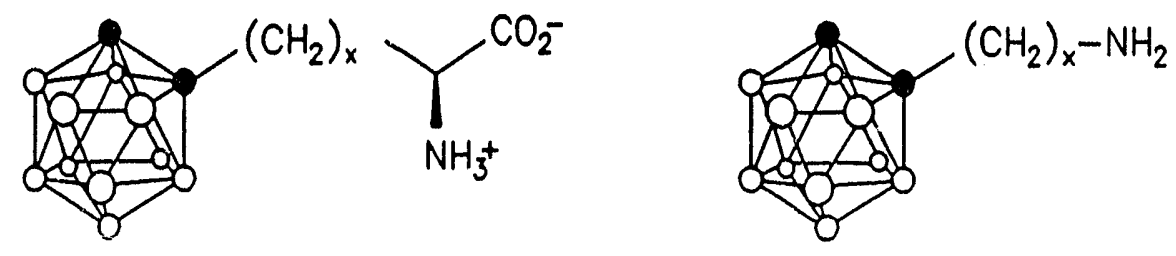


\section{Gadolinium NCT Agents}

We have synthesized and carried out biodistribution studies of gadolinium labeled liposomes (GLL) which were developed in our U.T. laboratory. Gadolinium (like boron-10) has an excellent neutron cross section and is considered to be of potential use in neutron capture therapy. GLL are constructed by adding gadolinium based amphiphiles, such as the one below, to liposome preparations.

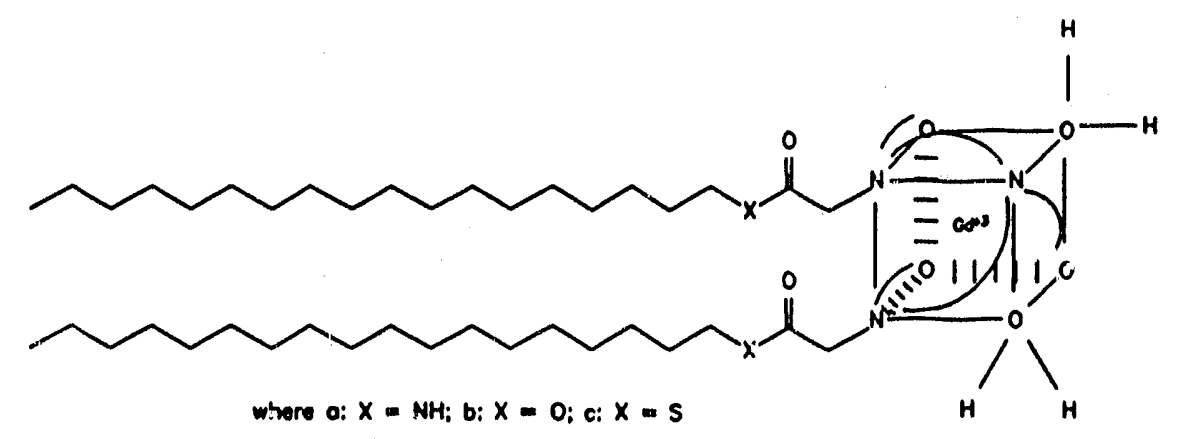

[See, for example, G. W. Kabalka, et al. Magn. Reson. Med., 8, 231 (1988).] Since liposomes can be selectively targeted to organs by coating them with various proteins or simply by size, we prepared a series of liposomes and investigated their retention in the livers of Balb/c mice. The GLL tested were constructed by linking the Gd-DTPA head group to the stearyl chains via amide (Gd-DTPA-SA), ester (Gd-DTPA-SE), and thioester (Gd-DTPA-ST) bonds. 


\section{B. MULTINUCLEAR MAGNETIC RESONANCE IMAGING}

Generation of MR images for fast relaxing nuclei such as boron is often hampered by the fact that the transverse relaxation times $\left(\mathrm{T}_{2}\right)$ are considerably shorter than the available time-to-echo (TE). The minimal TE achievable in MR imaging $i:$ iimited by the need to apply phase-encoding and dephasing/rephasing read-out gradients which have finite rise times imposed by the hardware limitations. Currently, echo times of $2.5 \mathrm{~ms}$ are attainable using conventional spin-warp imaging techniques and it may be possible to shorten TE to approximately $1 \mathrm{~ms}$ by reducing the resolution requirements. However, boron-11 and boron-10 possess intrinsic transverse relaxation rates in-vivo which are so short that even after $1 \mathrm{~ms}$ most of the signal has already decayed, resulting in an unacceptably low signal.

We modified an alternate imaging method designed by Elscint Corporation for nuclei with very short $T_{2}$ relaxation times. As in backprojection, the technique utilizes the FID, rather than the echo signal, but involves only phaseencoding of the signal without frequency encoding. The method was tested by obtaining boron- 11 images of aqueous boron phantoms, and was used to map the boron-11 distribution in an intact Fischer 344 rat infused with a therapeutic dose of dimeric sulfhydryl dodecaborane agent by out colleagues (Slatkin and Micca) at Brookhaven National Laboratory.

In the new method both spatial dimensions are phase-encoded by the usual amplitude cycling of gradients along the corresponding directions. The time 
during which these gradients are phase-encoding the signal is extremely short, 200 s. The time-domain signai is then Fourier transformed, in the usual manner, to yield an $x-y$ image. An extension to three spatial dimensions can be achieved by adding a third phase-encoding gradient along the z-axis.

The minimal performance time of the proposed sequence is longer than that of a conventional spin-warp sequence since at least one scan for each resolution element is required. But this is not an obstacle for the imaging of boron-11 in-vivo since the necessary number of scans needed for adequate signal averaging will usually exceed the minimal number of experiments required by the imaging process.

Imaging experiments were performed on a Gyrex $2 \mathrm{~T}, 90 \mathrm{~cm}$ bore, wholebody MR imaging unit operating at $26 \mathrm{MHz}$ for boron-11 using a 6-turn solenoidal coil (10 cm diameter) adjusted to the size of the rat. All imaging experiments were conducted at ambient temperatures. The animal used was a Fischer 344 male rate infused with sodium- $\mu$-disulfide-bis(undecahydro-closododecaborate), $\mathrm{Na}_{4} \mathrm{~B}_{24} \mathrm{H}_{22} \mathrm{~S}_{2}$, at Brookhaven National Laboratory; the rats were and shipped by air freight to Knoxville where the imaging experiments were performed.

The new method possesses a number of acivantages when compared to backprojection techniques. First, the natural linewidth of the signal (which is expected to be very broad due to the short $T_{2}$ ) does not degrade the spatial resolution since no frequency encoding is employed. Second, for the same 
reason, the images acquired by the method are free of artifacts and degradation resulting from magnetic field inhomogeneities, susceptibility variations and chemical shifts. (Both advantages are achieved without the need to employ gradients which are large compared to the prevalent interaction strengths in the spin system.) Third, the analog filter can be set to a much smaller frequency cutoff, compared to backprojection, which results in a substantial improvement in signal-to-noise.

\section{BIBLIOGRAPHY OF PUBLICATIONS}

\section{A. JOURNAL ARTICLES}

1. S. J. Kennel, S. Bultman, R. Lee, and G. W. Kabalka, "Rat Monoclonal Antibody Distribution in Mice: An Epitope Inside the Lung Vascular Space Mediates Very Efficient Localization", Int. J. Radiat. Appl. Instr. 17, 193 (1990).

2. P. Bendel, M. Davis, E. Berman, and G. W. Kabalka, "A Method for Imaging Nuclei with Short T2 Relaxation: Application to Boron-11 MR Imaging of a BNCT Agent in an Intact Rat," J. Mag. Reson., 88, 369 (1990).

3. G. W. Kabalka, "Gd-Labeled Liposomes Containing Amphipathic Agents for MRI", Invest. Radiol., in press. 
4. G. W. Kabalka, M. A. Davis, T. H. Moss, E. Buonocore, K. Hubner, E. Holmberg, K. Maruyama, and L. Huang, "Gadolinium-Labeled Liposomes Containing Various Amphiphilic Gd-DTPA Derivatives: 'Targeted MRI Contrast Enhancement Agents for the Liver", Magn. Reson. Med., in press.

5. G. W. Kabalka, M. A. Davis, E. Holmberg, K. Maruyama, and L. Huang, "Gadolinium-Labeled Liposomes Containing Amphiphilic GdDTPA Derivatives of Varying Chain Length: Targeted MRI Enhancement Agents", Magn. Reson. Imag., in press.

\section{B. INVITED PRESENTATIONS}

1. "Development of New Agents for Use in Modern Medical Imaging", Vanderbilt University, Nashville, Tennessee (March, 1990).

2. "Synthetic Approaches to Isotopically Labeled Compounds", University of California at Santa Cruz, Santa Cruz, California (March, 1990).

3. "Use of Organoboranes in Modern Medical Imaging", G. W. Kabalka, Boron USA II Workshop, Raleigh, NC (June, 1990).

4. "Isotope Incorporation via Organoborane Chemistry", International Symposium on Isotope Separation and Applications", Quebec, Canada (October, 1990). 
5. "Use of Organoboranes in the Design and Synthesis of Agents for Use in Modern Medical Imaging", Oesper Award Symposium, Cincinnati, Ohio (October, 1990).

6. "Design and Clinical Application of Agents for use in Medical Imaging," Chancellor's Associates, Knoxville, Tennessee (November, 1990).

7. "Synthesis of Pharmaceuticals Based on Organoborane Chemistry," University of New South Wales, Sydney, Australia (December, 1990).

8. "A New Boron MRI Method for Imaging BNCT Agents In Vivo", Fourth International Symposium on Neutron Capture Therapy for Cancer", University of Sydney, Sydney, Australia (December, 1990).

9. "Recent Advances in Magnetic Resonance Imaging: Organ Specific Relaxation Agents and Multinuclear Imaging of BNCT Agents", Royal Northshore Hospital, Sydney Australia (December, 1990).

\section{GRADUATE AND POSTDOCTORAL STUDENTS}

One postdoctoral student and three graduate students were supported during this period by the Department of Energy Nuclear Medicine Program.
A. Postdoctoral Student
Dr. Cuang-Qiang Cheng
B. Graduate Students
Mark A. Davis (Ph.D. candidate)
Genrge Hondrogiannis (Ph.D. candidate)
Zhe Wang (Ph.D. candidate)

\section{Page 10}

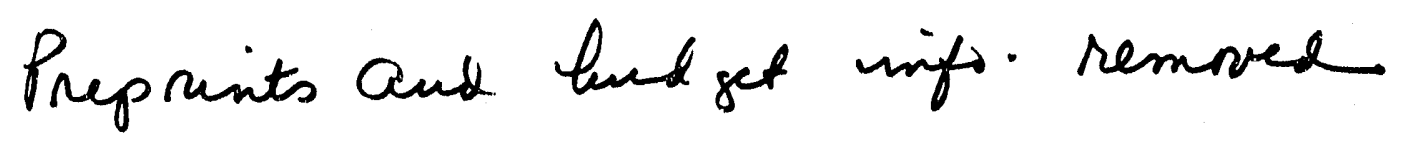



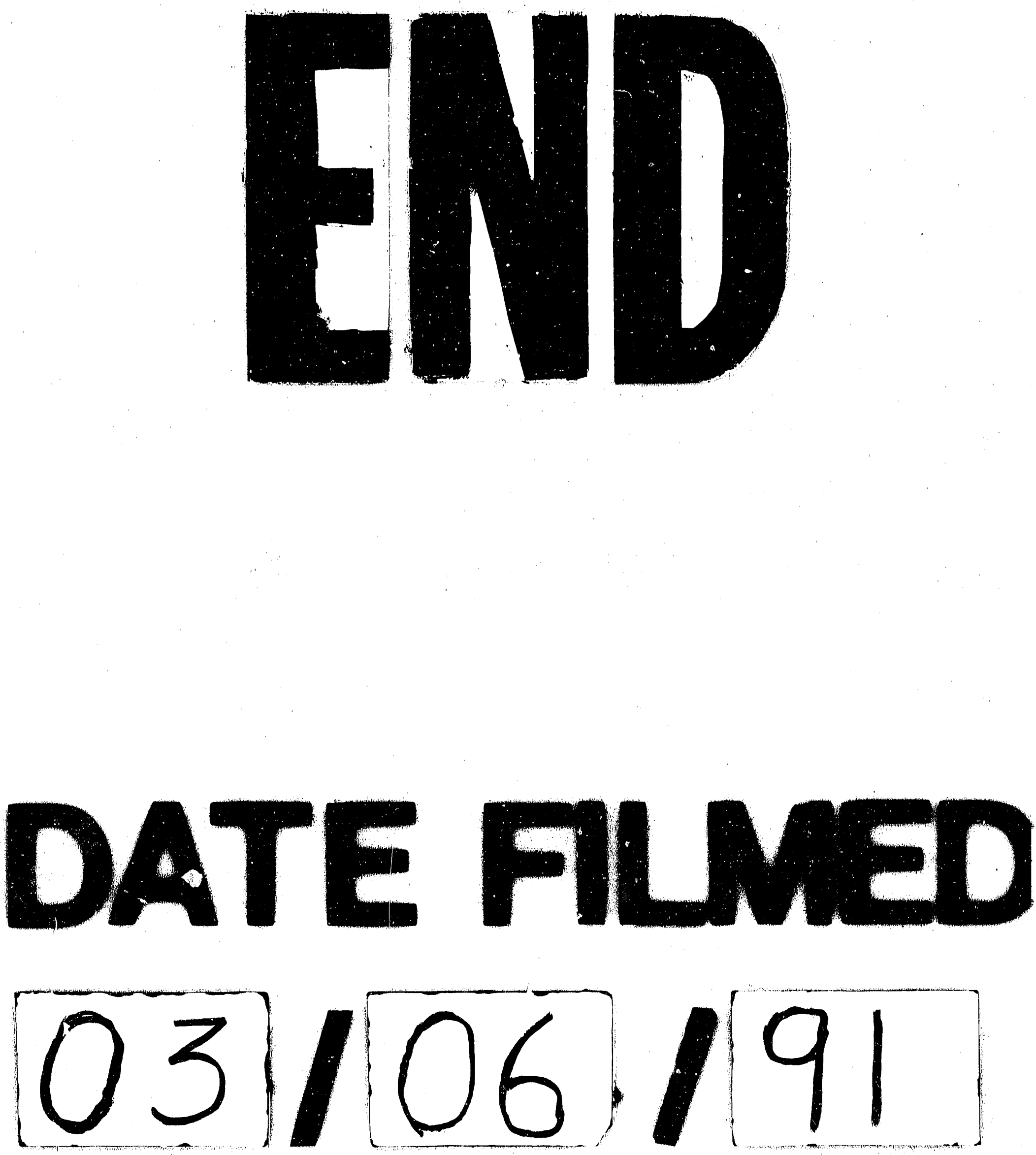
